\title{
The complexity of the set of squares in the homeomorphism group of the circle
}

\author{
by \\ Paul Gartside and Bojana Pejić (Pittsburgh, PA)
}

\begin{abstract}
The set of squares in the group of autohomeomorphisms of the circle is complete analytic, and hence analytic but not Borel.
\end{abstract}

1. Introduction. Polish groups are topological groups which are separable and completely metrizable. They are ubiquitous: Lie groups, separable Banach spaces, automorphism groups of first order structures (such as groups, fields, graphs etc.), and autohomeomorphism groups of manifolds, among others, are examples of Polish groups.

The key to unlocking a variety of problems in the theory of Polish groups is to determine which sets in a Polish group are definable both algebraically and topologically. By "algebraically definable" we mean sets such as the commutators, conjugacy classes, or the squares. In the context of Polish groups, "topologically definable" means being a Borel set. Intuitively a Borel set is one that can be defined from the open sets in countably many steps (a formal definition follows).

It is known that the set of squares $\left\{f^{2}=f \circ f \mid f \in H(I)\right\}$ in the autohomeomorphism group $H(I)$ of the closed unit interval $I$ is Borel. Indeed, it is clopen, as it is equal to the set of all order-preserving autohomeomorphisms of $I$ (see [4]). Remarkably, we show that if the endpoints of the unit interval are identified to form the circle, $S^{1}$, then the set of squares in its autohomeomorphism group $H\left(S^{1}\right)$ is (analytic but) not Borel. In fact, it is complete analytic.

The result that the squares in $H(I)$ are Borel is in contrast to the situation in the space $C(I, I)$ of all continuous maps from the unit interval into itself. By studying the properties of the set of points where a function is locally constant, Humke \& Laczkovich showed in [6] that the squares in

2000 Mathematics Subject Classification: 54H05, 03E15, 28A05.

Key words and phrases: Polish group, squares, Borel, complete analytic. 
$C(I, I)$ are (analytic but) not Borel. Later Beleznay improved this result by showing in [1] that the squares are complete analytic. Of course, autohomeomorphisms are nowhere locally constant. Instead, in proving that the squares in $H\left(S^{1}\right)$ are complete analytic, we focus on the structure of the set of fixed points.

Part of our interest in the topological definability of squares in Polish groups comes from Mackey's theorem [9] which says that a Polish group admits a unique Polish group topology provided there is a countable pointseparating collection of subsets which are Borel in every Polish group topology on the group. The most plausible candidates for the elements of the collection are algebraically defined sets. For example centralizers, $\{x \in G \mid$ $\left.x c x^{-1} c^{-1}=1\right\}=w^{-1}\{1\}$ where $w(x)=x c x^{-1} c^{-1}$, are necessarily closed (hence Borel) in every (Polish) group topology. In a compact group, forward images such as the squares, which we denote by $G^{2}=\left\{g^{2} \mid g \in G\right\}=w(G)$ where $w(x)=x^{2}$, and conjugacy classes are also necessarily closed. So one might anticipate that squares, commutators and so on would also be Borel in any Polish group. Indeed, in a general Polish group all conjugacy classes are Borel, and in an Abelian Polish group the squares are Borel. (These observations follow from the fact [10] that all orbits of a Polish group acting continuously on a Polish space are Borel: apply this to the conjugation action and the action $g \cdot x=2 g+x$, respectively.) But the example of the squares in the autohomeomorphism group of the circle dispels this hope. See [3] for further discussion of these topics.

The authors are grateful to one referee for bringing to their attention the following connections with ergodic theory. It was an old question of Halmos [5] whether each, say, weakly mixing transformation is a square of a transformation. This was answered in the negative by Chacon [2]. On the other hand, King has shown that the generic transformation has roots of all orders (see $[8]$ ).

Notation. We give definitions and some facts about Borel, analytic and complete analytic sets: all of these can be found in [7], which is our main reference for descriptive set theory. Let $X$ be a Polish (separable, completely metrizable) space. The set of Borel sets, $\mathbf{B}(X)$, is the smallest family of subsets of $X$ that contains the open sets and is closed under countable unions and taking complements. The Borel sets ramify into a hierarchy. In the first level are the open sets and the closed sets, in the second level the $G_{\delta}$ 's (countable intersections of open sets) and $F_{\sigma}$ 's (countable unions of closed sets), and in the third level the $F_{\sigma \delta}$ 's and $G_{\delta \sigma}$ 's. A subset $A$ of $X$ is analytic if it is the continuous image of a Polish space or, equivalently, of any Borel subset of a Polish space. Borel sets are analytic, but not vice versa. An analytic set $B$ of a Polish space $Y$ is complete if for any Polish space 
$X$ and any analytic set $A$ in $X$ there is a continuous function $F: X \rightarrow Y$ such that $F^{-1}(B)=A$. Such a function is called a continuous reduction of $A$ to $B$.

If $X$ is any set and $a_{1}, \ldots, a_{n}$ are distinct elements of $X,\left(a_{1} a_{2} \cdots a_{n}\right)$ denotes the permutation of $X$ that maps $a_{i}$ to $a_{i+1}$ for $i=1, \ldots, n-1$ and $a_{n}$ to $a_{1}$, leaving the other elements of $X$ fixed. We call such a permutation a finite cycle or, more specifically, an $n$-cycle. Similarly, for a sequence $\left(a_{i}\right)_{i \in \mathbb{Z}}$ of distinct elements of $X,\left(\begin{array}{llllll}\cdots & a_{-1} & a_{0} & a_{1} & a_{2} & \cdots\end{array}\right)$ denotes the permutation of $X$ that maps each $a_{i}$ to $a_{i+1}$ and leaves the other elements of $X$ fixed. Such a permutation is called an infinite cycle. Any permutation of $X$ can be represented by an unordered formal composition of disjoint cycles in a unique way. We say that a permutation $f$ "contains" a cycle $\sigma$ if $\sigma$ appears in the unique disjoint cycle representation of $f$.

2. Circular squares. Let $H\left(S^{1}\right)$ be the group of autohomeomorphisms of the unit circle $S^{1}$. Equipped with the topology of uniform convergence, it is a Polish group. We will show that the set

$$
H\left(S^{1}\right)^{2}=\left\{f^{2}=f \circ f \mid f \in H\left(S^{1}\right)\right\}
$$

of squares in $H\left(S^{1}\right)$ is complete analytic, and hence not Borel.

A standard technique for showing completeness of an analytic set is to reduce an already known complete analytic set to the given set. Beleznay [1] showed that the set LO2 of linear orders of type $I+I$ (a precise definition follows) is complete analytic. To show that $H\left(S^{1}\right)^{2}$ is complete, we construct in Lemma 2.3 a continuous reduction $F$ of LO2 to $H\left(S^{1}\right)^{2}$. Since LO2 is complete, an arbitrary analytic set $A$ can be reduced to it via a continuous map. Composing this reduction map with $F$ gives a continuous reduction of $A$ to $H\left(S^{1}\right)^{2}$. This proves that $H\left(S^{1}\right)^{2}$ is complete.

We first give in Lemma 2.2 a necessary and sufficient condition for a homeomorphism of $S^{1}$ of a certain type to be a square. Then we use this characterization to construct a continuous reduction of LO2 to $H\left(S^{1}\right)^{2}$ in Lemma 2.3.

We start by giving some definitions and notation. First, we recall the definition of the set LO2, as given in [1]. Let $\alpha \in 2^{\mathbb{N} \times \mathbb{N}}$ code the relation $R$ on $\mathbb{N}$ the following way:

$$
(n, m) \in R \quad \text { if and only if } \quad \alpha(n, m)=1 .
$$

Then LO is defined to be the set of those codes $\alpha \in 2^{\mathbb{N} \times \mathbb{N}}$ that code a linear order. LO is a $G_{\delta}$ subset of the Polish space $2^{\mathbb{N} \times \mathbb{N}}$, and thus a Polish space itself. For codes from LO we write $n<_{\alpha} m$ instead of $\alpha(n, m)=1$. The set $\mathrm{LO} 2$ is the collection of codes from LO which code a linear order of the form $I+I$ : 
LO2 $=\left\{\alpha \in \mathrm{LO} \mid \exists f \in 2^{\mathbb{N}}, g \in \mathbb{N}^{\mathbb{N}}\right.$ such that $g: \mathbb{N} \rightarrow \mathbb{N}$ is a bijection and: $\forall n, m \in \mathbb{N}, f(n)=0$ and $f(m)=1$ imply $n<_{\alpha} m ;$ $\forall n \in \mathbb{N}, f(n)=0$ if and only if $f(g(n))=1 ; \forall n \in \mathbb{N}, g(g(n))=n$; $\forall n, m \in \mathbb{N}, f(n)=f(m)=0$ imply that $n<_{\alpha} m$ iff $\left.g(n)<_{\alpha} g(m)\right\}$.

In other words, $f$ determines two classes of $\mathbb{N}$ such that every element of $f^{-1}(\{0\})$ is $\alpha$-less than every element of $f^{-1}(\{1\})$, and $g$ gives an $\alpha$-orderpreserving bijection of these two classes. As mentioned previously, LO2 is a complete analytic set.

While we visualize the topological space $S^{1}$ as the unit circle, formally we consider $S^{1}$ to be the quotient space obtained from the unit interval $I$ by identifying its endpoints 0 and 1 . For distinct points $x_{1}, \ldots, x_{n}(n \geq 3)$ in $S^{1}$ we write $x_{1}<\cdots<x_{n}\left(<x_{1}\right)$ if, when traveling anticlockwise along the circle $S^{1}$ starting from $x_{1}$, we reach points $x_{2}, x_{3}, \ldots, x_{n}$ in that order (before reaching $x_{1}$ again). For $x, y \in S^{1}$ we define the open interval $(x, y)=$ $\{z \mid x<z<y\}$. In the obvious way we also define " $\leq$ " and the closed and semi-closed intervals in $S^{1}$.

Let $H\left(S^{1}\right)^{+}$denote the set of order-preserving homeomorphisms of $S^{1}$ :

$$
H\left(S^{1}\right)^{+}=\left\{f \in H\left(S^{1}\right) \mid \forall x, y, z \in S^{1}, x<y<z \Rightarrow f(x)<f(y)<f(z)\right\} .
$$

Similarly, let $H\left(S^{1}\right)^{-}$be the collection of order-reversing homeomorphisms of $S^{1}$. Clearly $H\left(S^{1}\right)^{+}$is a subgroup of $H\left(S^{1}\right)$ of index 2, with $H\left(S^{1}\right)^{-}$as its other coset.

For $f \in H\left(S^{1}\right)^{+}$and $a, b \in S^{1}$ define $D(f \uparrow[a, b])=\left\{x \in[a, b] \mid f^{2}(x) \neq x\right\}$. of $S^{1}$ :

Finally, we define a collection $\mathcal{M}$ of a special kind of homeomorphisms

$$
\begin{aligned}
\mathcal{M}=\left\{f \in H\left(S^{1}\right)^{+} \mid\right. & f(0)=1 / 2, f(1 / 2)=1=0, \\
& \forall x \in(0,1 / 2), 0<x \leq f^{2}(x)<1 / 2 \text { and } \\
& \left.\forall x \in(1 / 2,1), 1 / 2<x \leq f^{2}(x)<1\right\} .
\end{aligned}
$$

Lemma 2.1. Let $f \in \mathcal{M}$. Suppose $0<a<a^{\prime}<c<c^{\prime}<1 / 2<b<b^{\prime}<$ $d<d^{\prime}<1$ are points in $S^{1}$ such that $f$ contains $(a b)(c d)\left(a^{\prime} b^{\prime}\right)\left(c^{\prime} d^{\prime}\right)$ in its disjoint cycle representation and for all $x \in\left(a, a^{\prime}\right) \cup\left(b, b^{\prime}\right) \cup\left(c, c^{\prime}\right) \cup\left(d, d^{\prime}\right)$, $f^{2}(x) \neq x$. Let $A=\left[a, a^{\prime}\right] \cup\left[b, b^{\prime}\right] \cup\left[c, c^{\prime}\right] \cup\left[d, d^{\prime}\right]$. Then there exists an orderpreserving homeomorphism $g$ of $A$ such that $g$ contains $(a c b d)\left(a^{\prime} c^{\prime} b^{\prime} d^{\prime}\right)$ and $f\left\lceil A=g^{2}\right.$.

Proof. Fix an arbitrary point $a_{0,0} \in\left(a, a^{\prime}\right)$ and for $n \in \mathbb{Z}$, let $a_{n, 0}=$ $f^{2 n}\left(a_{0,0}\right) \in\left(a, a^{\prime}\right)$, and $b_{n, 0}=f^{2 n+1}\left(a_{0,0}\right) \in\left(b, b^{\prime}\right)$. For $r \in(0,1)$ and $n \in \mathbb{Z}$, let $a_{0, r}=a_{0,0}+r\left(a_{1,0}-a_{0,0}\right), a_{n, r}=f^{2 n}\left(a_{0, r}\right) \in\left(a, a^{\prime}\right)$, and $b_{n, r}=$ $f^{2 n+1}\left(a_{0, r}\right) \in\left(b, b^{\prime}\right)$. Starting with an arbitrary point $c_{0,0}$ in $\left(c, c^{\prime}\right)$, construct analogous sequences $\left(c_{n, r}\right)$ and $\left(d_{n, r}\right)$ in $\left(c, c^{\prime}\right)$ and $\left(d, d^{\prime}\right)$ respectively. It is not hard to show that the following statements hold for the sequence $\left(a_{n, r}\right)$ : 
(i) $a<\cdots<a_{-1,0}<a_{0,0}<a_{1,0}<a_{2,0}<\cdots<a^{\prime}$.

(ii) $a_{n, 0} \rightarrow a$ as $n \rightarrow-\infty$ and $a_{n, 0} \rightarrow a^{\prime}$ as $n \rightarrow \infty$.

(iii) $a<a_{n, r}<a_{m, s}<a^{\prime}$ if and only if $n+r<m+s$.

(iv) For all $x \in\left(a, a^{\prime}\right)$, there are $n \in \mathbb{Z}$ and $r \in[0,1)$ such that $x=a_{n, r}$.

The analogous statements hold for the sequences $\left(b_{n, r}\right),\left(c_{n, r}\right)$ and $\left(d_{n, r}\right)$. The disjoint cycle decomposition of $f \nmid A$ is then

$$
(a b)(c d)\left(a^{\prime} b^{\prime}\right)\left(c^{\prime} d^{\prime}\right) \cdot \prod_{r \in[0,1)}\left(\cdots a_{0, r} b_{0, r} a_{1, r} b_{1, r} \cdots\right)\left(\cdots c_{0, r} d_{0, r} c_{1, r} d_{1, r} \cdots\right) .
$$

It is clear from the construction that every cycle in this composition is a cycle of $f\lceil A$. But (iv) also implies that every cycle of $f\lceil A$ is included in this representation. Define $g$ to be the permutation of $A$ given by the following disjoint cycle representation:

$$
(a c b d)\left(a^{\prime} c^{\prime} b^{\prime} d^{\prime}\right) \cdot \prod_{r \in[0,1)}\left(\cdots a_{0, r} c_{0, r} b_{0, r} d_{0, r} a_{1, r} c_{1, r} b_{1, r} d_{1, r} \cdots\right) .
$$

Then clearly $g^{2}=f \nmid A$. That $g$ is order-preserving follows from (iii). The inverse images under $g$ of the open intervals in $S^{1}$ are open intervals because $g$ is an order-preserving bijection. Thus $g$ is continuous.

Lemma 2.2 (Characterization of squares in $\mathcal{M}$ ). For a homeomorphism $f \in \mathcal{M}$ the following are equivalent:

(i) $f \in H\left(S^{1}\right)^{2}$.

(ii) There exist $c \in(0,1 / 2)$ and an order-preserving homeomorphism $\phi:[0, c] \rightarrow[c, 1 / 2]$ such that $\phi(D(f\lceil[0, c]))=D(f \uparrow[c, 1 / 2])$.

Proof. Suppose that $f \in H\left(S^{1}\right)^{2}$ and let $g \in H\left(S^{1}\right)$ be such that $f=g^{2}$. Note that $g(0) \neq 0$, otherwise $f(0)=g^{2}(0)=0$. Also, $g(0) \neq 1 / 2$, for otherwise $f(1 / 2)=f(g(0))=g^{3}(0)=g(f(0))=g(1 / 2)=g(g(0))=f(0)=$ $1 / 2$. We claim that $g \in H\left(S^{1}\right)^{+}$. Suppose, for a contradiction, $g \in H\left(S^{1}\right)^{-}$. If $0<g(0)<1 / 2<0$, then applying $g$ to each of these points reverses the order of their images, i.e., $g(0)>1 / 2>g(1 / 2)>g(0)$. In particular, $g(1 / 2) \in$ $(0,1 / 2)$. Now applying $g$ again, we find $1 / 2<g(1 / 2)<0<1 / 2$. However, this gives $g(1 / 2) \in(1 / 2,1)$, a contradiction! The case $0<1 / 2<g(0)<0$ yields a contradiction in a similar way. So $g$ must indeed be order-preserving. Now, either $g(0) \in(0,1 / 2)$ or $g^{-1}(0) \in(0,1 / 2)$. In case $g(0) \in(0,1 / 2)$, let $c=g(0)$ and let $\phi=g \uparrow[0, c]$. Then $\phi$ is an order-preserving homeomorphism of $[0, c]$ with $[c, 1 / 2]$ and for $x \in[c, 1 / 2]$ we have

$$
\begin{aligned}
x \in \phi(D(f \uparrow[0, c])) & \Leftrightarrow g^{-1}(x) \in D\left(f\lceil[0, c]) \Leftrightarrow f^{2}\left(g^{-1}(x)\right) \neq g^{-1}(x)\right. \\
& \Leftrightarrow g^{3}(x) \neq g^{-1}(x) \Leftrightarrow g^{4}(x) \neq x \Leftrightarrow f^{2}(x) \neq x \\
& \Leftrightarrow x \in D(f\lceil[c, 1 / 2]),
\end{aligned}
$$


so $\phi\left(D(f\lceil[0, c]))=D(f \uparrow[c, 1 / 2])\right.$. In case $g^{-1}(0) \in(0,1 / 2)$, the proof is similar, only with $c=g^{-1}(0)$ and $\phi=g^{-1} \uparrow[0, c]$.

Conversely, suppose that (ii) holds. Let $C=\left\{x \in[0, c] \mid f^{2}(x)=x\right\}$. Note that $0, c \in C$. Define $g_{C}$ on $A=C \cup \phi(C) \cup f(C) \cup f(\phi(C))$ by the following disjoint cycle representation:

$$
\prod_{x \in C}(x \phi(x) f(x) f(\phi(x))) .
$$

Then $g_{C}$ is an order-preserving homeomorphism of $A$ and $g_{C}^{2}=f\lceil A$. The set $D(f \uparrow[0, c])$ consists of disjoint open intervals. Let $D$ be a component of $D(f \uparrow[0, c])$. Let $g_{D}$ be the order-preserving homeomorphism of $B=\bar{D} \cup$ $\overline{\phi(D)} \cup \overline{f(D)} \cup \overline{f(\phi(D))}$ such that $f\left\lceil B=g_{D}^{2}\right.$, constructed in Lemma 2.1. Define

$$
g=\bigcup\left\{g_{D} \mid D \text { is a component of } D(f \uparrow[0, c])\right\} \cup g_{C} .
$$

It is not hard to check that then $g$ is a well-defined order-preserving homeomorphism of $S^{1}$ with $f=g^{2}$.

LEMma 2.3. There is a continuous function $F: \mathrm{LO} \rightarrow \mathcal{M}$ such that $F(\alpha) \in H\left(S^{1}\right)^{2}$ if and only if $\alpha \in \mathrm{LO} 2$.

Proof. Fix $\alpha \in \mathrm{LO}$. We want to define $F(\alpha) \in \mathcal{M}$. We start by constructing a discrete collection of open intervals $\left\{\left(p_{n}, q_{n}\right) \mid n \in \mathbb{N}\right\}$ with endpoints in $(0,1 / 2)$ and with the following properties:

(a) The order of $\left\{p_{n} \mid n \in \mathbb{N}\right\}$ is isomorphic to the order coded by $\alpha$;

(b) $\inf \left\{p_{n} \mid n \in \mathbb{N}\right\}=0$ if and only if the order has no smallest element;

(c) $\sup \left\{q_{n} \mid n \in \mathbb{N}\right\}=1 / 2$ if and only if the order has no largest element;

(d) For any $x \notin \bigcup_{n \in \mathbb{N}}\left(p_{n}, q_{n}\right), \sup \left\{q_{n} \mid q_{n} \leq x\right\}=\inf \left\{p_{n} \mid p_{n} \geq x\right\}$ if and only if there is no biggest $q_{n}$ below $x$ and no smallest $p_{n}$ above $x$;

(e) $\left|q_{n}-p_{n}\right|<1 / n$.

To do this, we follow the construction of Beleznay in [1]. Let $\mathcal{O}=\left\{\left(a_{m}, b_{m}\right) \mid\right.$ $m \in \mathbb{N}\}$ be an enumeration of the rational open subintervals with endpoints in $(0,1 / 2)$. Choose a pairwise disjoint subsystem of $\mathcal{O}$ as follows. Let $\left(s_{1}, t_{1}\right)=\left(a_{1}, b_{1}\right), t_{0}=0$ and $s_{0}=1 / 2$. Assume that we have already chosen $\left(s_{k}, t_{k}\right)$ for $k=1, \ldots, n-1$ such that if $k<_{\alpha} l$ then $t_{k}<s_{l}$ (i.e., $\left(s_{k}, t_{k}\right)$ precedes $\left.\left(s_{l}, t_{l}\right)\right)$. Let $i$ be the $\alpha$-biggest among $1, \ldots, n-1$ that is $\alpha$-less than $n$, if such an $i$ exists. Otherwise let $i=0$. Let $j$ be the $\alpha$-smallest among $1, \ldots, n-1$ that is $\alpha$-bigger than $n$, and if no such $j$ exists, let $j=0$. By the choice of $s_{k}, t_{k}$ for $k=0,1, \ldots, n-1$, we see that $t_{i}<s_{j}$. Let $\left(s_{n}, t_{n}\right)$ be the first $\left(a_{m}, b_{m}\right)$ which

(i) is strictly inside $\left(t_{i}, s_{j}\right)$,

(ii) contains $\left(t_{i}+s_{j}\right) / 2$, and

(iii) $\left|b_{m}-a_{m}\right|<1 / n$. 
It is clear that this process can be continued and yields a pairwise disjoint system of intervals $\left\{\left(s_{n}, t_{n}\right) \mid n \in \mathbb{N}\right\}$ such that the order of $\left\{s_{n} \mid n \in \mathbb{N}\right\}$ is isomorphic to the order coded by $\alpha$. We now let $\left(p_{n}, q_{n}\right)$ be the middle third of the interval $\left(s_{n}, t_{n}\right)$. The collection $\left\{\left(p_{n}, q_{n}\right) \mid n \in \mathbb{N}\right\}$ constructed this way has all of the aforementioned properties: (b), (c), (d) are ensured by (ii), and (e) is implied by (iii).

Let $U=\bigcup_{n \in \mathbb{N}}\left(p_{n}, q_{n}\right)$. We now define $F(\alpha)$ as follows: for $t \in S^{1}=$ $[0,1] / \sim$, let

$$
F(\alpha)(t)=\left\{\begin{array}{lc}
1 / 2+t & \text { if } t \in[0,1 / 2) \backslash U, \\
1 / 2+t+\frac{q_{n}-p_{n}}{\pi} \sin \left(\frac{\pi}{q_{n}-p_{n}}\left(t-p_{n}\right)\right) & \text { if } t \in\left(p_{n}, q_{n}\right), \\
-1 / 2+t & \text { if } t \in[1 / 2,1) \backslash(1 / 2+U), \\
-1 / 2+t+\frac{q_{n}-p_{n}}{\pi} \sin \left(\frac{\pi}{q_{n}-p_{n}}\left(t-1 / 2-p_{n}\right)\right) & \text { if } t \in 1 / 2+\left(p_{n}, q_{n}\right) .
\end{array}\right.
$$

If we visualize $S^{1}$ as the unit circle, then $F(\alpha)$ acts on the points outside $U$ and $1 / 2+U$ as the rotation by $\pi$, while each point in $U$ and $1 / 2+U$ is rotated by "a little over" $\pi$. More precisely, a point $t$ in $\left(p_{n}, q_{n}\right)$ is taken to a point in $1 / 2+\left(p_{n}, q_{n}\right)$ between the point $1 / 2+t$ diametrically opposite to $t$ and $1 / 2+q_{n}$. Similarly, a point $t$ in $1 / 2+\left(p_{n}, q_{n}\right)$ is taken to a point in $\left(p_{n}, q_{n}\right)$ between its diametrically opposite point $-1 / 2+t$ and $q_{n}$. It is easy to see that $F(\alpha) \in \mathcal{M}$.

We show that $F: \mathrm{LO} \rightarrow \mathcal{M}$ is continuous. For $\alpha \in \operatorname{LO}$ let $\left\{\left(p_{n}^{\alpha}, q_{n}^{\alpha}\right)\right.$ $n \in \mathbb{N}\}$ be the discrete collection of open intervals constructed from $\alpha$. Fix $\alpha \in \mathrm{LO}$. We show that $F$ is continuous at $\alpha$. Specifically, we prove that for all $\varepsilon>0$, there is an open neighborhood $N_{\alpha}$ of $\alpha$ such that for all $\beta \in N_{\alpha}$, $d(F(\alpha), F(\beta))<\varepsilon$.

Let $\varepsilon>0$. Let $n_{\varepsilon}=\lceil 1 / \varepsilon\rceil$. Then, by (e), for all $\beta \in$ LO, $n \geq n_{\varepsilon} \Rightarrow$ $\left|q_{n}^{\beta}-p_{n}^{\beta}\right|<\varepsilon$. Let

$$
N_{\alpha}=\left\{\beta \in \mathrm{LO} \mid \beta \text { and } \alpha \text { agree on the order of } 1, \ldots, n_{\varepsilon}-1\right\} .
$$

This is a basic open neighborhood of $\alpha$. If $\beta \in N_{\alpha}$, then the intervals $\left(p_{n}^{\alpha}, q_{n}^{\alpha}\right)$ and $\left(p_{n}^{\beta}, q_{n}^{\beta}\right)$ coincide for $n<n_{\varepsilon}$, so $F(\alpha)$ and $F(\beta)$ may differ only on the set

Thus,

$$
A=\bigcup_{n \geq n_{\varepsilon}}\left(p_{n}^{\alpha}, q_{n}^{\alpha}\right) \cup \bigcup_{n \geq n_{\varepsilon}}\left(p_{n}^{\beta}, q_{n}^{\beta}\right)
$$

$$
\begin{aligned}
d(F(\alpha), F(\beta)) & =\sup \left\{|F(\alpha)(t)-F(\beta)(t)| \mid t \in S^{1}\right\} \\
& =\sup \{|F(\alpha)(t)-F(\beta)(t)| \mid t \in A\} .
\end{aligned}
$$

If $t \in A$, then $t \in\left(p_{n}^{\alpha}, q_{n}^{\alpha}\right)$ for some $n \geq n_{\varepsilon}$, or $t \in\left(p_{m}^{\beta}, q_{m}^{\beta}\right)$ for some $m \geq \varepsilon$, 
or both. If $t$ belongs to $\left(p_{n}^{\alpha}, q_{n}^{\alpha}\right)$ and no other component of $A$, then

$$
|F(\alpha)(t)-F(\beta)(t)| \leq\left|q_{n}^{\alpha}-p_{n}^{\alpha}\right|<\varepsilon .
$$

Similarly, if $t$ belongs to $\left(p_{m}^{\beta}, q_{m}^{\beta}\right)$ only, then

$$
|F(\alpha)(t)-F(\beta)(t)| \leq\left|q_{m}^{\beta}-p_{m}^{\beta}\right|<\varepsilon .
$$

Finally, if $t \in\left(p_{n}^{\alpha}, q_{n}^{\alpha}\right) \cap\left(p_{m}^{\beta}, q_{m}^{\beta}\right)$, then

$$
|F(\alpha)(t)-F(\beta)(t)| \leq \max \left(\left|q_{n}^{\alpha}-p_{n}^{\alpha}\right|,\left|q_{m}^{\beta}-p_{m}^{\beta}\right|\right)<\varepsilon .
$$

Thus, $d(F(\alpha), F(\beta))<\varepsilon$. This proves the continuity of $F$.

We now turn to proving $F(\alpha) \in H\left(S^{1}\right)^{2} \Leftrightarrow \alpha \in \mathrm{LO} 2$.

Suppose $F(\alpha) \in H\left(S^{1}\right)^{2}$. Then by Lemma 2.2, there exist $c \in(0,1 / 2)$ and an order-preserving homeomorphism $\phi:[0, c] \rightarrow[c, 1 / 2]$ such that

$$
\phi(D(F(\alpha) \uparrow[0, c]))=D(F(\alpha) \uparrow[c, 1 / 2]) .
$$

Then $c \notin U$, for otherwise $c \in D(F(\alpha) \uparrow[0, c])$, and so $\phi(c) \in D(F(\alpha) \uparrow[c, 1 / 2])$, which further implies that $\phi(c) \in U \cup(1 / 2+U)$. But $\phi(c)=1 / 2 \notin U \cup$ $(1 / 2+U)$.

Since $D(F(\alpha) \uparrow[0,1 / 2])=U$, we find that

$$
\phi\left(\bigcup_{p_{n} \in(0, c)}\left(p_{n}, q_{n}\right)\right)=\bigcup_{p_{n} \in(c, 1 / 2)}\left(p_{n}, q_{n}\right) .
$$

Since $\phi$ is a homeomorphism, if $p_{n} \in(0, c)$, then $\phi\left(\left(p_{n}, q_{n}\right)\right)$ is equal to $\left(p_{m}, q_{m}\right)$ for some $m$ with $p_{m} \in(c, 1 / 2)$. This further means that for $p_{n} \in$ $(0, c), \phi\left(p_{n}\right)=p_{m}$ for some $p_{m} \in(c, 1 / 2)$.

Let $J=\left\{n \mid p_{n} \in(0, c)\right\}$ and $K=\left\{n \mid p_{n} \in(c, 1 / 2)\right\}$. Then, in $(0,1 / 2)$, every element of $J$ precedes every element of $K$ and $\psi: J \rightarrow K$ defined by

$$
\psi(n) \text { is the unique } m \text { such that } \phi\left(p_{n}\right)=p_{m}
$$

is an order-preserving bijection between $J$ and $K$. Thus, the order of $\left\{p_{n} \mid\right.$ $n \in \mathbb{N}\}$ is of the form $I+I$. Since this order is isomorphic to the order coded by $\alpha$, we see that $\alpha \in \mathrm{LO} 2$.

Conversely, suppose $\alpha \in \mathrm{LO} 2$. According to Lemma 2.2, we need to find $c \in(0,1 / 2)$ and an order-preserving homeomorphism $\phi:[0, c] \rightarrow[c, 1 / 2]$ with the property $\phi(D(F(\alpha) \uparrow[0, c]))=D(F(\alpha) \uparrow[c, 1 / 2])$.

Write $\mathbb{N}$ as the disjoint union of sets $J$ and $K$ such that each element of $J$ is $\alpha$-less than each element of $K$ and there is an $\alpha$-order-preserving bijection $\tau: J \leftrightarrow K$. Let $c_{1}=\sup \left\{q_{n} \mid n \in J\right\}$ and $c_{2}=\inf \left\{p_{n} \mid n \in K\right\}$. If $J$ has no biggest element, let $c=c_{1}$. If $K$ has no smallest element, let $c=c_{2}$. (This definition of $c$ is not ambiguous, since in the case that both $J$ has no biggest element and $K$ has no smallest element, $c_{1}=c_{2}$ by (d).) Otherwise, let $c=\left(c_{1}+c_{2}\right) / 2$. Notice that if $J$ has a biggest element then $c_{1}<c$, and if $K$ has a smallest element then $c<c_{2}$. 
Define $\phi\left(p_{n}\right)=p_{\tau(n)}$ and $\phi\left(q_{n}\right)=q_{\tau(n)}$. Then $\phi$ is strictly order-preserving on the set $A=\left\{p_{n}, q_{n} \mid n \in J\right\}$.

Extend $\phi$ to the closure of $A$ as follows. First note that if $x \in \bar{A}$, then either $x=\sup \left\{q_{n} \mid q_{n} \leq x\right\}$ or $x=\inf \left\{p_{n} \mid p_{n} \geq x\right\}$ (or both). In case $x=\sup \left\{q_{n} \mid q_{n} \leq x\right\}$, define $\phi(x)=\sup \left\{q_{\tau(n)} \mid q_{n} \leq x\right\}$. In case $x=\inf \left\{p_{n} \mid\right.$ $\left.p_{n} \geq x\right\}$, define $\phi(x)=\inf \left\{p_{\tau(n)} \mid p_{n} \geq x\right\}$. This is well-defined (nonambiguous): Suppose $x=\sup \left\{q_{n} \mid q_{n} \leq x\right\}=\inf \left\{p_{n} \mid p_{n} \geq x\right\}$. We need to show that $y:=\sup \left\{q_{\tau(n)} \mid q_{n} \leq x\right\}$ and $z:=\inf \left\{p_{\tau(n)} \mid p_{n} \geq x\right\}$ are equal. Note that $y=\sup \left\{q_{m} \mid q_{m} \leq y\right\}=\sup \left\{q_{m} \mid q_{m} \leq z\right\}$ and $z=\inf \left\{p_{m} \mid p_{m} \geq z\right\}$. By property $(\mathrm{d})$, there is no biggest $q_{n}$ below $x$ and there is no smallest $p_{n}$ above $x$. Since $\tau$ is an order-preserving bijection, it follows that there is no biggest $q_{m}$ below $y$, and thus no biggest $q_{m}$ below $z$, and also, there is no smallest $p_{m}$ above $z$. This, by (d) again, implies $\sup \left\{q_{m} \mid q_{m} \leq z\right\}=\inf \left\{p_{m} \mid\right.$ $p_{m} \geq z$, i.e., $y=z$. Clearly $\phi$ is continuous and order-preserving on $\bar{A}$.

Note that

$$
\begin{aligned}
\inf A=0 & \Leftrightarrow J \text { has no smallest element (by (b)) } \\
& \Leftrightarrow K \text { has no smallest element } \\
& \Leftrightarrow \inf \tau(A)=c \text { (by the definition of } c), \\
\sup A=c & \Leftrightarrow J \text { has no biggest element (definition of } c) \\
& \Leftrightarrow K \text { has no biggest element } \\
& \Leftrightarrow \inf \tau(A)=1 / 2(\text { by }(\mathrm{c})) .
\end{aligned}
$$

Next we define $\phi(0)=c$ and $\phi(c)=1 / 2$. By the above remarks, $\phi$ is welldefined (i.e., if $\phi$ has already been defined at 0 and/or $c$, this new definition agrees with the previous one) and $\phi$ is order-preserving and continuous on $\bar{A} \cup\{0, c\}$.

The complement of $\bar{A} \cup\{0, c\}$ in $[0, c]$ is a disjoint union of open intervals. We define $\phi$ to be linear on each of these to get an order-preserving homeomorphism of $[0, c]$ and $[c, 1 / 2]$.

Of course,

$$
\phi(D(F(\alpha) \uparrow[0, c]))=\phi\left(\bigcup_{n \in J}\left(p_{n}, q_{n}\right)\right)=\bigcup_{m \in K}\left(p_{m}, q_{m}\right)=D(F(\alpha) \uparrow[c, 1 / 2]) .
$$

Theorem 2.4. $H\left(S^{1}\right)^{2}$ is a complete analytic set.

Proof. The set $H\left(S^{1}\right)^{2}$ is obviously analytic, since $f \mapsto f \circ f$ is continuous. Lemma 2.3 gives a continuous reduction of LO2, a complete analytic set, to $H\left(S^{1}\right)^{2}$. Hence $H\left(S^{1}\right)^{2}$ is complete.

\section{References}

[1] F. Beleznay, The complexity of the collection of countable linear orders of the form $I+I$, J. Symbolic Logic 64 (1999), 1519-1526. 
[2] R. V. Chacon, A geometric construction of measure preserving transformations, in: Proc. Fifth Berkeley Sympos. on Math. Statist. Probab., Vol. II: Contributions to Probability Theory, Univ. of California Press, Berkeley, CA, 1967, Part 2, 335-360.

[3] P. Gartside and B. Pejić, Uniqueness of Polish group topologies, submitted.

[4] M. Gerstenhaber, On canonical constructions, II, Proc. Nat. Acad. Sci. U.S.A. 42 (1956), 881-883.

[5] P. R. Halmos, Lectures on Ergodic Theory, Chelsea, Bronx, NY, 1960.

[6] P. D. Humke and M. Laczkovich, The Borel structure of iterates of continuous functions, Proc. Edinburgh Math. Soc. 32 (1989), 483-494.

[7] A. S. Kechris, Classical Descriptive Set Theory, Springer, New York, 1995.

[8] J. L. King, The generic transformation has roots of all orders, Colloq. Math. 84/85 (2000), 531-547.

[9] G. W. Mackey, Borel structures in groups and their duals, Trans. Amer. Math. Soc. 85 (1957), 134-165.

[10] D. E. Miller, On the measurability of orbits in Borel actions, Proc. Amer. Math. Soc. 63 (1977), 165-170.

Department of Mathematics

University of Pittsburgh

301 Thackeray Hall

Pittsburgh, PA 15260, U.S.A.

E-mail: gartside@math.pitt.edu

bop4+@pitt.edu

Received 15 June 2006;

in revised form 7 May 2007 